\title{
Poor Kidney Function and the risk of Prostate Cancer among Aged Men in Kogi East, Kogi State, Nigeria
}

\author{
Zainab Yahaya', Adam Abdullahi Adam², Asma'u Mahe², \\ Obidoa Onyechi ${ }^{3}$ and Sanni Momoh ${ }^{1}$ \\ ${ }^{1}$ Biochemistry Department, \\ Kogi State University Ayingba, \\ Kogi State Nigeria \\ ${ }^{2}$ Biochemistry Department, \\ Federal University Dutse, \\ PMB 7156, \\ Dutse Jigawa State Nigeria \\ ${ }^{3}$ Biochemistry Department, \\ University of Nigeria, \\ Nsukka
}

Email:adam.a@fud.edu.ng

\begin{abstract}
Prostate Specific Antigen (PSA) is a protein that is produced by cells in the male prostate gland. An increase in PSA levels is associated with prostate inflammation, prostate cancer, and benign prostatic hypertrophy. This work provides information on the values of Kidney function indices and PSA in apparently normal ageing men residing within the Eastern zone of Kogi State, Nigeria. The subjects were divided into different groups based on their age range. There was a gradual increased in PSA and serum urea as the age increases.
\end{abstract}

Keyword: Kidney, Prostate cancer, Prostate Specific Antigen, Blood Urea Nitrogen and Creatinine

\section{INTRODUCTION}

The risk factors for chronic kidney disease (CKD) (eg, hypertension and diabetes) increase in prevalence in the elderly, and the effect of the risk factors is cumulative over many years. As a result, there has been a tremendous increase in the number of elderly patients with CKD and end-stage kidney disease. Kidney (Renal) function is an indication of the state of the kidney and its role in renal physiology (Stevens et al., 2006). Blood urea nitrogen and creatinine will not be raised above the normal value until $60 \%$ of total kidney function is lost. There is the need to check creatinine clearance rate whenever kidney disease is suspected. High creatinine level points to kidney disease. Low urea nitrogen concentrations were alluded to over hydration, starvation, and alcohol (Gifford and Leel-Khouri, 1989).

Prostate cancer is the most common malignancy among elderly men and is the second leading malignancy in the Western world (Crawford, 2003). The incidence of prostate cancer 
has steadily increased over the last decade (Crawford, 2003; Jemal et al., 2007). Between 2000 and 2050, the number of men over 65 years is expected to increase 4 -fold worldwide. By 2030 , the percentage of men older than 65 years will rise to $19.6 \%$ of the population compared with $12.4 \%$ population in 2000 (Crawford, 2003; Jemal et al., 2007). Thus, the percentage of men who will be diagnosed with prostate cancer and those who will require treatment for their malignancy will rise in the coming years.

Apparent Risk Factors for Prostate Pathology includes; western life style/smoking/alcohol/diet, Probability increases if father had it, incident increases with age, too much and very little sex, high blood pressure, stimulants such as caffeine affects the static and dynamic functions of prostate, red meat, lack of Vitamin D, high calcium intake, cold temperatures (e.g. Air condition), cycling (pressure on the perineum can inflames the prostate), Cadmium containing water (in oil producing areas) (Obidoa, 2007). Prostate Specific Antigen (PSA) is a protein that is produced by cells in the male prostate gland. An increase in PSA levels is associated with prostate inflammation, prostate cancer, and benign prostatic hypertrophy (Simmons et al., 2011).

\section{MATERIALS AND METHODS}

\section{Experimental Design}

The study was carried out in Kogi east comprising of Idah, Dekina and Ankpa local government areas. Blood samples were collected from volunteered donors (men) aged 25-75 years. Questionnaire was used to collect the demographic data from the subjects for analysis. Sixty five questionnaires were given out; five were considered invalid due to errors in filling the form by the respondents. Ten (10) questionnaires were given to control subjects from people aged 25 - 34 years old. All the questionnaires were accepted and used as being valid for the analysis.

\section{Sample Preparation}

Blood samples were collected from the volunteered donors; men aged 25years and above through venipuncture, into EDTA coated tubes and plain tubes for the biochemical test. The samples were centrifuged at $4000 \mathrm{rpm}$ for 5 minutes using uniscope laboratory centrifuge model SM 112 to obtain the serum required for analysis.

\section{Determination of serum creatinine (direct endpoint procedure)}

This was done according to the method of Cook (1975). The working reagent was prepared by combining equal volumes of creatinine Picric Acid Reagent and Creatinine Buffer Reagent and mixed well. The test vial, reagent blank, standard, controls and unknown test tubes were labeled. Three (3.0) $\mathrm{ml}$ of working reagent was pipetted into all test tubes. Samples $(0.1 \mathrm{ml})$ was pipetted into their respective tubes, distilled water to reagent blank and mixed. All tubes were incubated in water bath at $37^{\circ} \mathrm{C}$ for 15 minutes. The spectrophotometer was set at the wavelength of $510 \mathrm{~nm}$ and zeroed with the reagent blank. The absorbance of all tubes was read.

\section{Determination of serum urea nitrogen (colorimetric method)}

This was done according to the method of Veniamin and Vakirtzi-Lemania (1970). The BUN enzyme regent was reconstituted by adding $100 \mathrm{ml}$ distilled water and mixed well. The test vial, reagent blank, Standard, controls and unknown test tubes were labeled. BUN enzyme reagent $(1.5 \mathrm{ml})$ was pipetted into labeled test tubes and allowed to equilibrate to room temperature. Sample $(0.01 \mathrm{ml})$ was added to its respective test tube and mixed gently, using deionized water as the sample for the reagent blank. All the test tubes were incubated in 
water bath at $37^{\circ} \mathrm{C}$ for five (5) minutes. BUN $(1.5 \mu l)$ color Developer was added to the test tubes and mixed gently. The test tubes were incubated for five (5) minutes at $37 \mathrm{oC}^{\mathrm{C}}$. The spectrophotometer was zeroed with the reagent blank at $630 \mathrm{~nm}$. The absorbance of the tubes were read and recorded.

\section{Determination of Prostate specific antigen (PSA)}

This was done according to the method of ACS, (2007). The test kit and acetate buffer were brought to room temperature. The test device was placed on a clean and level surface. Serum $(40 \mu \mathrm{l})$ was pipetted and dispensed into the sample well and a drop of acetate buffer was added to the sample well of the test kits immediately. Results were taken after 5 minutes following the formation of the color lines that appeared on the test kits.

\section{Statistical Analysis}

Data was initially sorted out manually and tabulated and then entered into the computer using bonferon comparison test of graph pad with statistical software and the mean value determined, and the differences were considered significant if $p<0.05$. Comparison of mean values in relation to age distribution was done using analysis of variance (ANOVA).

\section{RESULTS}

Variation of the urea concentration with age

Figure 1 shows increases in urea concentration $(p<0.05)$ in the test groups 2 and 3 , and a non- significant difference in groups 4 and 5 and then rises again in group 6 (75- above).

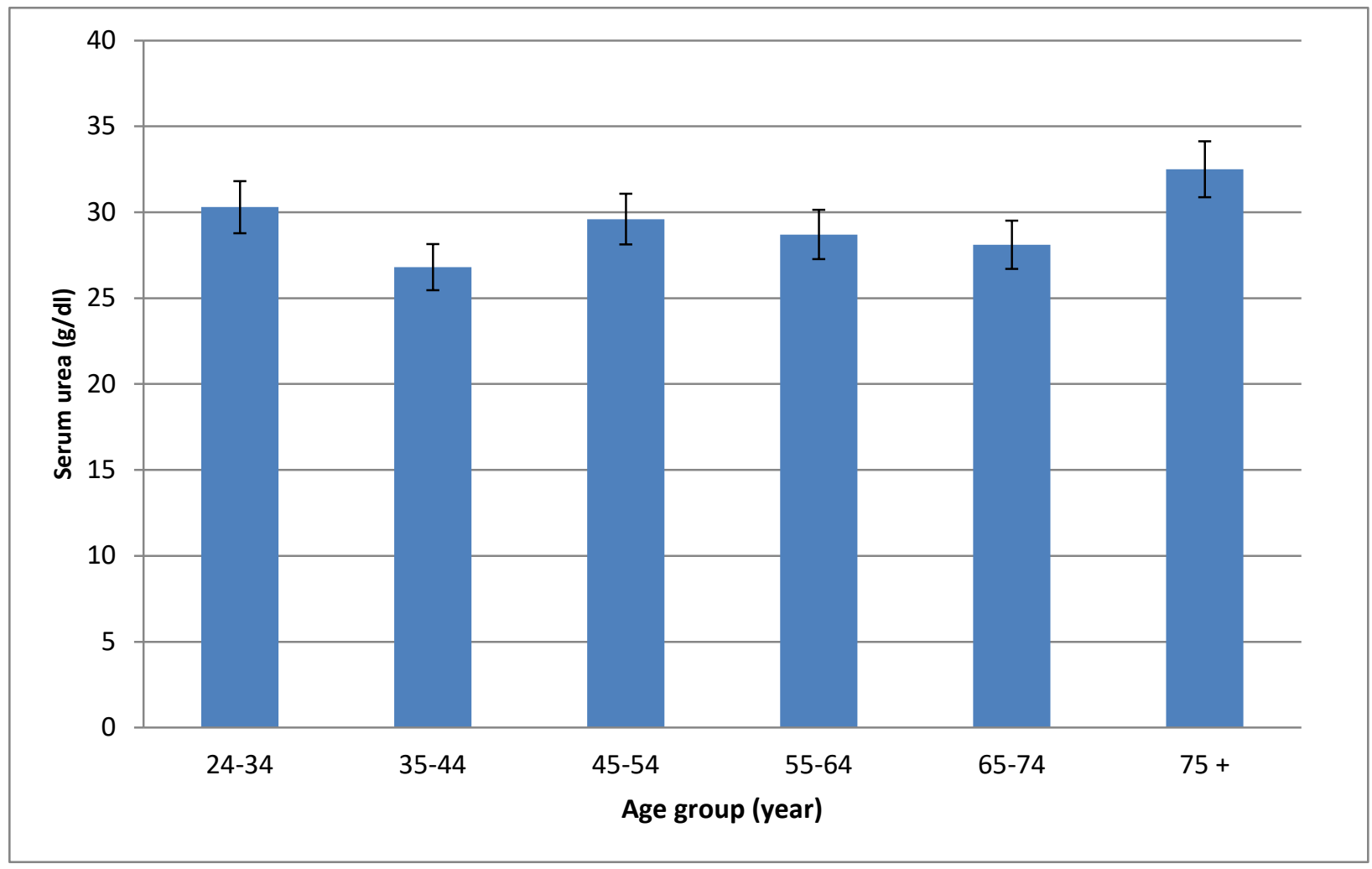

Fig. 1: Bar chart showing Serum urea with age, $n=60$

Variation of the serum creatinine with age

In fig. 2, there is elevation of serum creatinine in the test groups 2, 3 and 4 when compared with the control group 1 while a decrease occurred in groups 5 and 6 , though not significant. 


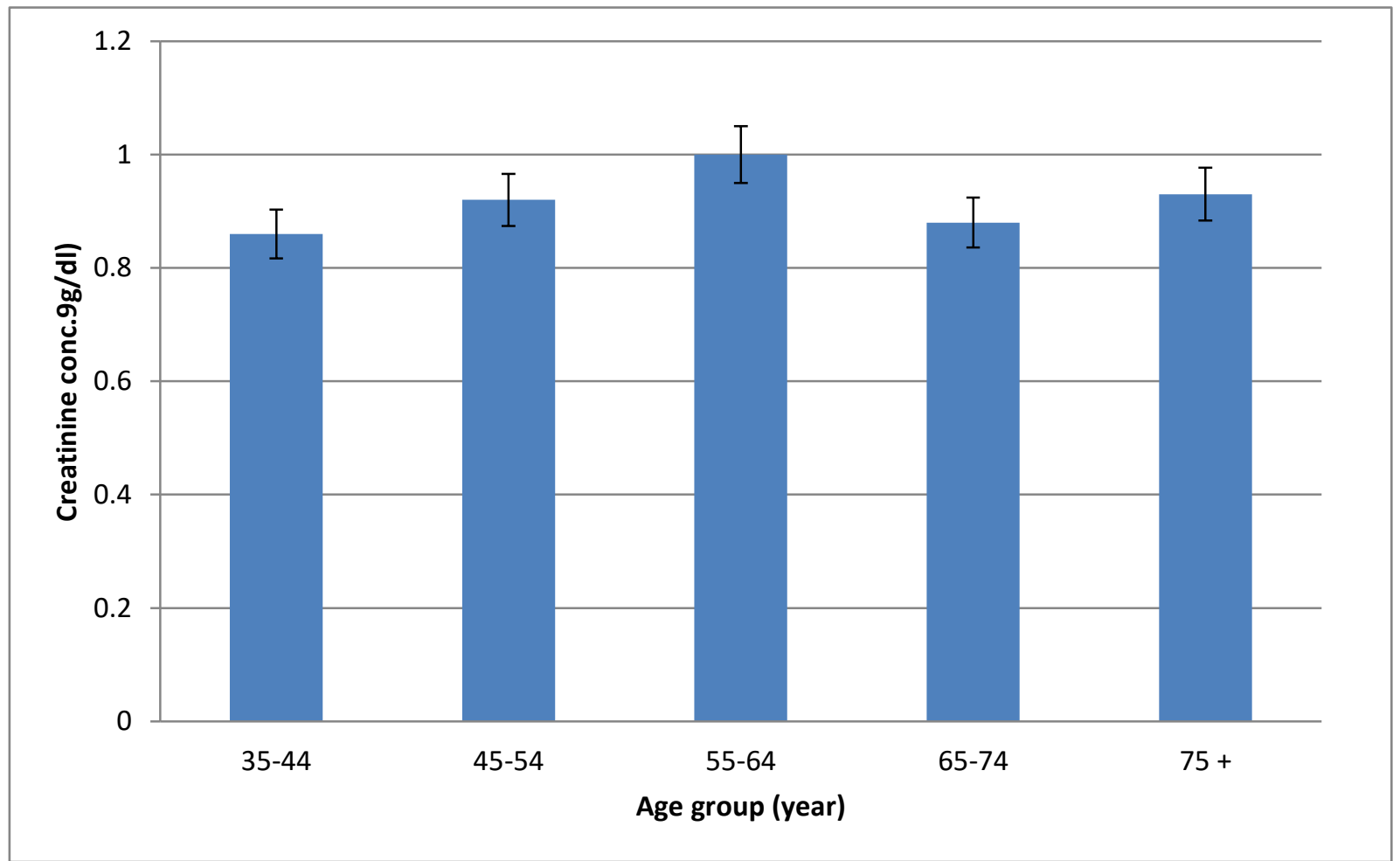

Fig.2: Bar chart showing creatinine with age, $n=60$

\section{Variation of the prostate specific antigen (PSA) with age}

Figure 3 shows increases in PSA in all groups when compared with control group 1 and standard reference value, indicating an increase in PSA as the age increases. These increases especially test group 5 and 6 is an indication that the subjects are at risks of prostatitis; an inflammation of the prostate glands.

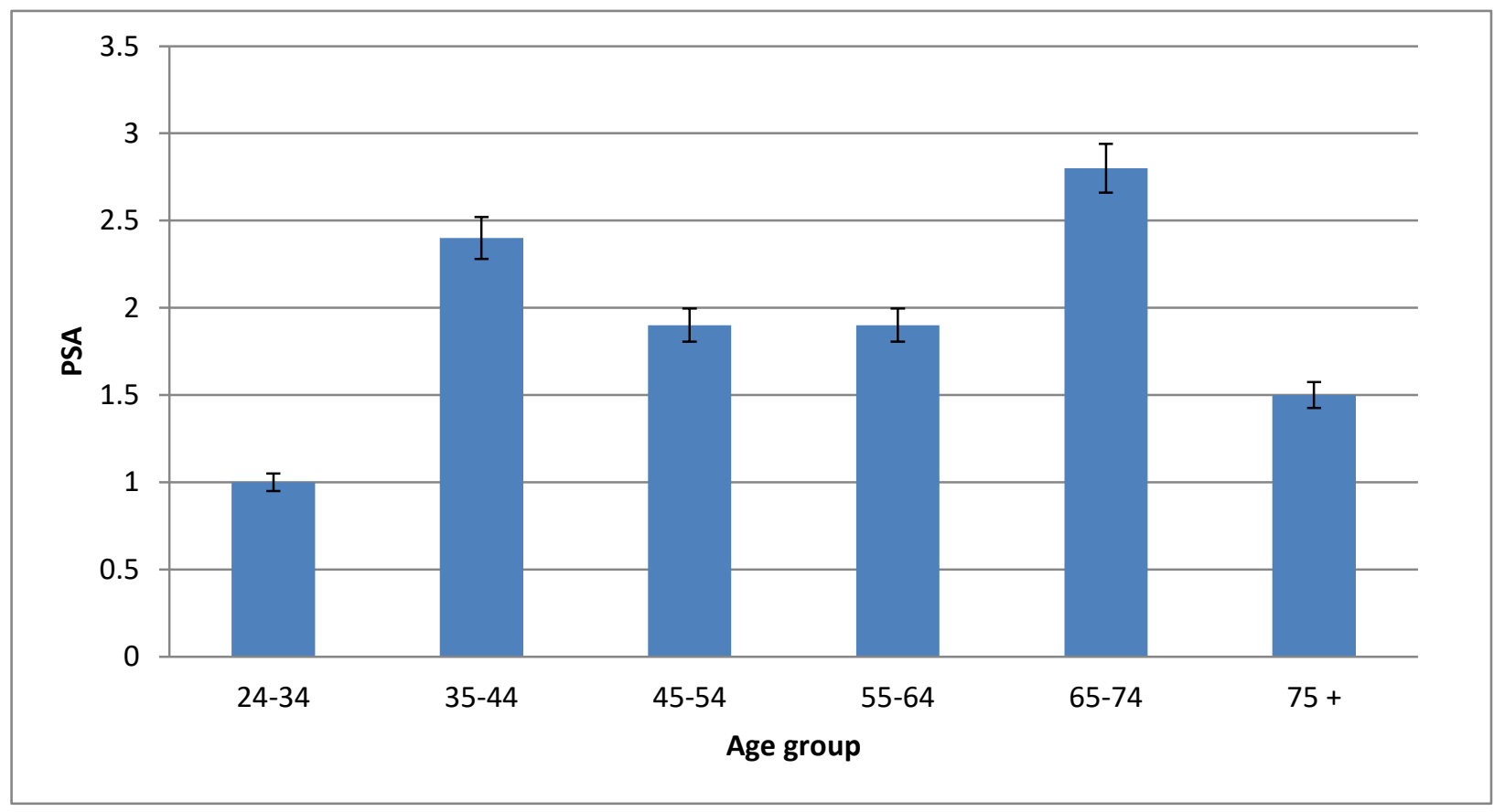

Fig.3: Bar chart showing prostate specific antigen with age, $n=60$ 


\section{DISCUSSION}

The risk factors for chronic kidney disease (CKD) (eg, hypertension and diabetes) increase in prevalence in the elderly, and the effect of the risk factors is cumulative over many years. As a result, there has been a tremendous increase in the number of elderly patients with CKD and end-stage kidney disease. Older age, hypertension, diabetes, anemia, hyperuricemia, hyperhomocysteinemia, hypertriglyceridemia, obesity, and LDL-Cholesterol may be accountable for higher prevalence of CKD. Three studies reported the estimations of CKD prevalence among adults 18 years or older were 11.8\% (Shanghai) (Chen et al., 2009), 13\% (Beijing) (Zhang et al., 2008), and 19.1\% (Tibet) (Chen et al., 2011), respectively. In Guangzhou, $12.1 \%$ residents 20 years or older had at least one indicator of kidney damage. In Beijing, $11.3 \%$ adults older than 40 years had CKD (Zhang et al., 2007).

The prostate specific antigen (PSA) showed a significant increase in group 5 (65-74) years and group 6 (75 and above) when compared to the control group 1 and standard reference value range. The serum factor prostate specific antigen and lipid profile were chosen for analysis during the study because they are suggested to be linked with prostate pathologies (Farnsworth et al., 1981; Femini and Barett-Connor, 1998). These increases indicate that the subjects were at risk of prostatitis. Age plays a vital role in prostate cancer treatment decisions. Elderly men with high-risk prostate cancer are usually under-treated because they are offered fewer and less effective choices of treatment compared to younger men that may result in higher rates of cancer mortality in this group, according to a study at University of California San Francisco (UCSF) (Bechis et al., 2011).

\section{References}

American Cancer Society. Cancer Facts \& Figures 2007. Atlanta, GA: American Cancer Society, 2007.

Bechis, S.K., Carroll, P.R., and Cooperberg, M.R. (2011). Impact of age at diagnosis on prostate cancer treatment and survival. J Clin Oncol; 29: 235-41

Chen, N., Wang,W., Huang, Y., Shen, P., Pei, D., Yu, H., Shi, H., Zhang, Q., Xu, J., and Lv, Y.(2009). Community-based study on CKD subjects and the associated risk factors. Nephrol. Dial. Transplant. 24, 2117-2123. [CrossRef]

Chen, W., Liu, Q., Wang, H., Chen, W., Johnson, R., Dong, X., Li, H., Ba, S., Tan, J., and Luo, N.(2011). Prevalence and risk factors of chronic kidney disease: A population study in the Tibetan population. Nephrol. Dial. Transplant. 26, 1592-1599.

Crawford, E.D.(2003). Epidemiology of prostate cancer. Urology; 62(6 suppl 1):3-12.

Farnsworth, W. E, Slaanwhite, W..R., Sharma, M, Oseko, F; Brown, Gonder M. and Cartagena, R.(1981). Interaction of prolactin and testosterone in the human prostate. Urol. Res 9:79-88

Femini, R. L. and Barret-connor. E. (1998). Sex hormones and Age: A cross sectional study of testosterone and estradiol and their bio available factions in Community- dwelling men American Journal of Epidemiology 147 (8) 750-754

Gifford, L. and Leel-Khouri, S. (1989). Significance of low serum urea nitrogen concentrations. Clinical Chemistry, 35 (4): 639 - 640.

Jemal, A., Siegel, R., Ward, E.( 2007). Cancer statistics. CA Cancer J Clin; 57:43-66.

Obidoa, O. (2007). Inaugural lecture University of Nigeria Nsukka

Simmons, M. N., Berglund RK and Jones JS. (2011). A Practical Guide to Prostate Cancer Diagnosis and Management. Cleaveland Clinic Journal of Medicine. 78: 321

Stevens, L. A., Coresh, J., Greene, T. and Levey, A. S. (2006). Assessing kidney function measured and estimated glomerular filtration rate. The New England Journal of Medicine, 354 (23): 2473 - 2483. 
Zhang, L., Zhang, P., Wang, F., Zuo, L., Zhou, Y., Shi, Y., Li, G., Jiao, S., Liu, Z., and Liang, W.(2008). Prevalence and factors associated with CKD: A population study from Beijing. Am. J. Kidney Dis. 51, 373-384. [CrossRef]

Zhang, L., Zuo, L., Xu, G., Wang, F., Wang, M., Wang, S., Lv, J., Liu, L., and Wang, H.(2007). Community-based screening for chronic kidney disease among populations older than 40 years in Beijing. Nephrol. Dial. Transplant. 22, 1093-1099. [CrossRef] [PubMed] 